Article

\title{
Radiosensitizer effect of $\beta$-apopicropodophyllin against colorectal cancer via induction of reactive oxygen species and apoptosis
}

\author{
Na-Gyeong Lee ${ }^{1,2,}$, , Jin-Hee Kwon ${ }^{1,}{ }^{,}$,A-Ram Kang ${ }^{1}$, Jie-Young Song ${ }^{1}$, Sang-Gu Hwang ${ }^{1}$, Hong-Duck Um ${ }^{1}$, \\ Joon Kim ${ }^{2,+ \text { and Jong Kuk Park }}{ }^{1,+}$
}

1 Division of Radiation Biomedical Research, Korea Institute of Radiological and Medical Sciences, Seoul 01812, Korea; ilr27387@naver.com (N.-G.L.); arbam0919@kirams.re.kr (A.-R.K.); peterjane19canada@gmail.com(J.-H.K); immu@kirams.re.kr (J.-Y.S.); sgh63@kirams.re.kr (S.-G.H.); hdum@kirams.re.kr (H.-D.U.);jkpark@kirams.re.kr(J.K. P)

2 Division of Life Sciences, Korea University, Seoul 02841, Republic of Korea; joonkim@korea.ac.kr (J.K.)

* These authors performed experiments equally

† Co-Correspondence: jkpark@kirams.re.kr; Tel.: +82-02-970-1321, joonkim@korea.ac.kr

\begin{abstract}
APP), a derivative of podophyllotoxin (PPT), has been identified as a potential anti-cancer drug. This study tested whether APP acts as an anti-cancer drug and can sensitize colorectal cancer (CRC) cells to radiation treatment. APP had an anti-cancer effect against the CRC cell lines HCT116, DLD-1, SW480 and COLO320DM with IC50 values of $7.88 \mathrm{nM}, 8.22 \mathrm{nM}, 9.84 \mathrm{nM}$ and $7.757 \mathrm{nM}$, respectively induction of DNA damage. Colonogenic and cell counting assays indicated that the combined treatment of APP and $\gamma$-ionizing radiation (IR) showed greater retardation of cell growth than either alone, suggesting that APP sensitizes CRC cells to IR. Annexin V-propidium iodide (PI) assays and immunoblot analysis showed that the combined treatment of APP and IR increased apoptosis in CRC cells compared with either APP or IR alone. Results obtained from the xenograft experiments also indicated that the combination of APP and IR enhanced apoptosis in in vivo animal model. Apoptosis induction by the combined treatment of APP and IR resulted from reactive oxygen species (ROS). Inhibition of ROS by N-acetylcysteine (NAC) restored cell viability and decreased the induction of apoptosis by APP and IR in CRC cells. Taken together, these results indicate that a combined treatment of APP and IR might promote apoptosis by inducing ROS in CRC cells.
\end{abstract}

Keywords: $\beta$-Apopicropodophyllin; Radiosensitizer; Topoisomerase inhibitor; ROS; Apoptosis; Colorectal cancer

\section{Introduction}

Colorectal cancer (CRC is the third most common type of cancer in the United States, with almost 150,000 patients newly diagnosed with CRC in 2020. Although usually occurring in individuals aged $>50$ years, about $12 \%$ of newly diagnosed patients and $7 \%$ of deaths from CRC have been reported in individuals aged $<50$ years $[1,2]$. The treatment modalities for CRC include surgery, radiation therapy, and systemic treatments such as chemotherapy, immunotherapy, and targeted therapy such as anti-EGFR (epidermal growth factor receptor) agent cetuximab and the anti-angiogenesis agent bevacizumab. Most patients with CRC are diagnosed with regional or distant metastasis, requiring additional 
chemotherapy after surgery or palliative chemotherapy for metastatic cancer [3, 4]. Traditional chemotherapy agents and radiotherapy for CRC not only affect the tumor but also non-target organs, resulting in the development of adverse effects or resistance [5]. Resistance is a major obstacle to cancer treatment, being responsible directly or indirectly for over $80 \%$ of deaths; therefore, novel drugs and treatment strategies are required to overcome tumor resistance to current treatments.

Podophyllotoxin acetate (PA) is a natural compound that induces cancer cell death by disturbing microtubule stability and inducing cell cycle arrest, ER stress and autophagy, making it as a candidate for novel cancer drug development [6]. PA is a chemical agent related to podophyllotoxin (PPT) that isolated from Podophyllum peltatum Linnaeus, and shown to have antiviral affects against herpes, measles, and influenza viruses, as well as against venereal warts and skin cancer. PPT has been involved in the development of semi-synthetic anti-cancer derivatives, such as etoposide, teniposide and etopophos, which act by inhibiting DNA topoisomerases [7]. DNA topoisomerases are one of the major chemotherapeutic targets and there were continuous efforts to develop novel antibacterials and anticancer agents $[8,9]$. The topological modifications related to DNA transcription and replication are controlled by various topoisomerases through several mechanisms such as introducing negative or positive supercoils into DNA, catenating or decatenating circular and linear DNA, and relaxing positive or negatively supercoiled DNA [10,11]. There are three categories of molecular mechanisms for topoisomerase inhibition. One of them are competitive inhibition to substrates that inhibitor binds directly to active site of topoisomerase. The other mechanisms include competitive inhibition on the ATP binding site and blocking the enzyme activity by inducing ternary protein-DNA-inhibitor complex formation [12-16]. Among these inhibitory mechanisms, topoisomerase-targeted chemotheray is postulated as clinically significant events because through this mechanism, the enzyme is forced to form cleavage complex causing cell death [17]. Another topoisomerase-targeted chemotheray include the redox-productive effect and the induction of covalent bond formation in the drug-enzyme complex, which is followed by cell death. [18]. These topoisomerase inhibitor-derived anti-cancer drugs, such as irinotecan and etoposide are used for chemotherapy of several cancers including non-small cell lung cancer (NSCLC) and colorectal cancer (CRC). [19-21]. $\beta$-apopicropodophyllin (APP) is a derivative of PPT that disturbs microtubule polymerization and induces apoptosis of NSCLC cells such as NCI-H460, NCI-H1299, A549, and their xenografts in nude mice in vivo models, suggesting that it may be a candidate anti-cancer agent in our previous studies [22,23]. To prove anti-cancer effect of APP extended from these previous findings, anti-cancer or radiosensitizing effect of APP against CRC cells in vitro and in vivo were evaluated in the present study.

\section{Results}

\subsection{APP induces the death of CRC cells by inducing DNA damage}

Previously, APP (Fig. 1A) was synthesized from PPT and shown to have anti-cancer and radiosensitizing effects against NSCLC cells in vitro and in vivo $[17,18]$. This study tested whether APP could also induce cell death and have radiosensitization effects against two CRC cell lines, HCT116 and DLD-1. The IC50 values of APP after 48 and $72 \mathrm{~h}$ were calculated to be $9.79 \mathrm{nM}$ and $8.51 \mathrm{nM}$, respectively, in HCT116 cells and $9.27 \mathrm{nM}$ and $8.41 \mathrm{nM}$, respectively, in DLD-1 cells (Fig. 1B-E). We also detected IC50 values of APP for additional CRC cell lines - SW480 and COLO320DM. IC50 values of APP after 48 and $72 \mathrm{~h}$ are following as; SW480 are $13.78 \mathrm{nM}$ and $9.84 \mathrm{nM}$, respectively, and COLO320DM are 
$8.28 \mathrm{nM}$ and $7.76 \mathrm{nM}$, respectively. These IC50 values for various CRC cells were used as standard doses in the following experiments, and implied APP might be an effective anti-cancer drug treating colorectal cancer. Because HCT-116 cells contain wild type $p 53$ and DLD- 1 cells contain mutant p53 [24, 25], the similar IC50 values in these cell lines indicate that the cytotoxicity of APP is not related to p53 status. To confirm the anti-cancer effect of APP against CRC cells, immunoblot analysis was performed using $\gamma \mathrm{H} 2 \mathrm{AX}$ antibody. APP treatment was found to increase the expression of $\gamma \mathrm{H} 2 \mathrm{AX}$ in CRC cells (Fig. 1C), suggesting that the anti-cancer effect of APP is due to its induction of DNA damage.

A<smiles>COc1cc(C2=C3C(=O)OC=C3Cc3cc4c(cc32)OCO4)cc(OC)c1OC</smiles>

$\beta$-apopicropodophyllin (APP)

D

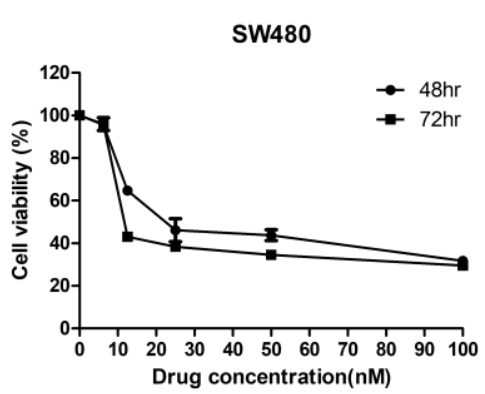

B

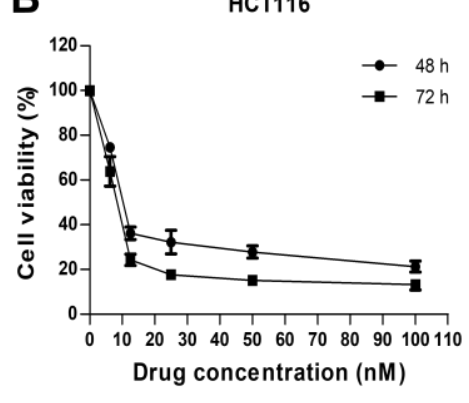

$\mathbf{E}$

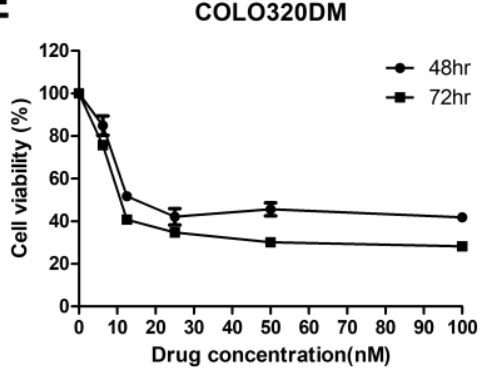

C
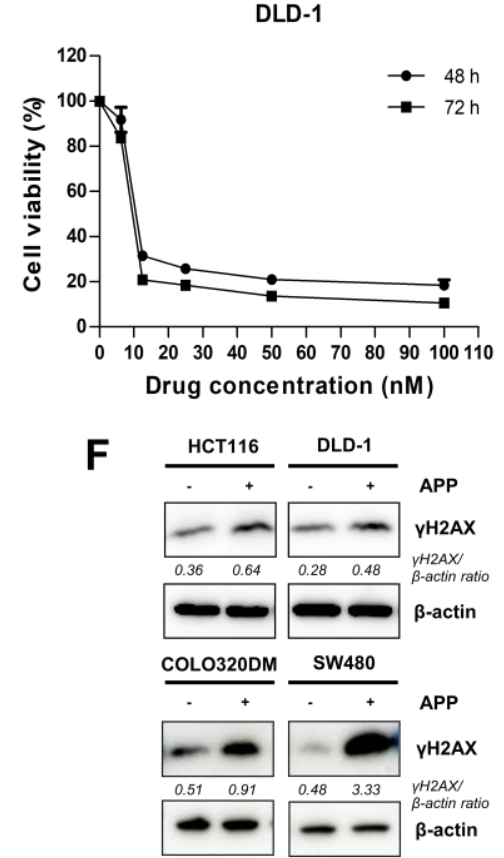

Figure 1. Anti-cancer effects of APP on human colorectal cancer cells. (A) Chemical structure of APP. (B-E) IC50 values determination of APP. CRC cell lines HCT116, DLD-1, SW480 and COLO320DM cells were treated with $6.25,12.5,25,50$ or $100 \mathrm{nM}$ APP for 48 or $72 \mathrm{~h}$, and cell viability measured by MTT assays. IC50 values for APP against CRC cell lines were calculated as described in the Materials and Methods. (F) Immunoblot assays for detection of $\gamma \mathrm{H} 2 \mathrm{AX}$ activation in cells treated with APP. HCT116, DLD-1, SW480 and COLO320DM cells were incubated with or without $7.5 \mathrm{nM}$ APP for $24 \mathrm{~h}$ prior to harvesting.

\subsection{APP acts as a radiosensitizer by retarding cell growth in vitro}

The radiosensitization effects of APP against HCT116 and DLD-1 cells were tested in clonogenic assays. We used HCT116 and DLD-1 to test p53-independent radiosensitizing effect of APP, because HCT116 cell line contains wild type $p 53$ gene, but DLD-1, SW480 and COLO320DM are mutant $p 53$ gene [26]. Cells were pre-treated with $7.5 \mathrm{nM}$ APP for $16 \mathrm{~h}$, followed by IR with 1, 2, 3, or 4 Gy and culture of the cells for 14 days. Clonogenic assays showed that the surviving fraction of cells treated with APP and IR was lower than the surviving fraction of cells treated with IR alone (Fig. 2A). Based on a survival fraction of 0.1 , the dose-enhancement ratios (DERs) for HCT-116 and DLD-1 cells were calculated to be 1.13 and 1.31, respectively (Table 1). More than 1 of DER values indicated APP enhanced radiation effects. Additionally, cell counting assays were performed to assess the effects of the combined treatment of 7.5 nM APP and 3 Gy IR on cell death (Fig. 2B-2E). Cell death rates were over 1.5 2-fold higher in both HCT-116, DLD-1, SW480 and COLO320DM cells treated with APP and IR than in cells treated with APP or IR alone (Fig. 2B-2E). In addi- 
tion, immunoblot analyses with anti- $\gamma \mathrm{H} 2 \mathrm{AX}$ antibody showed that the combined treatment of APP and IR enhanced $\gamma \mathrm{H} 2 \mathrm{AX}$ expression in both cell lines compared with single treatment of APP or IR alone (Fig. 2F). Collectively, these results indicate that APP can induce radio-sensitization CRC cells possibly by inducing DNA damage which leads to anti-cancer effect.
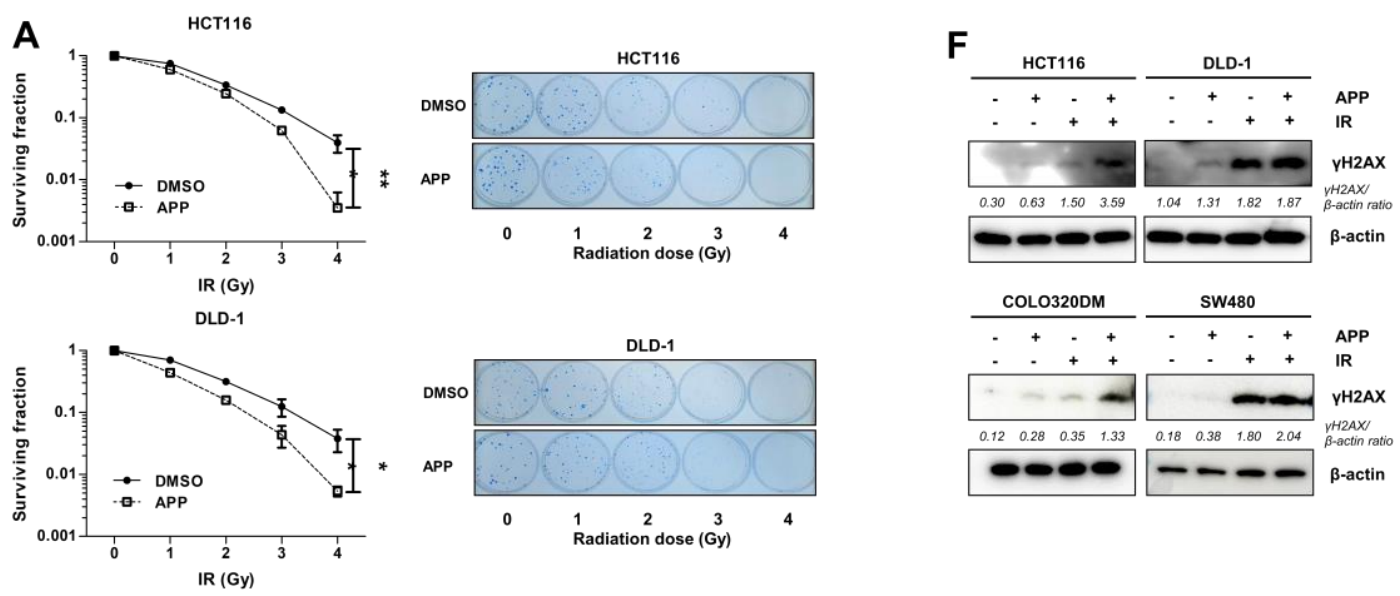

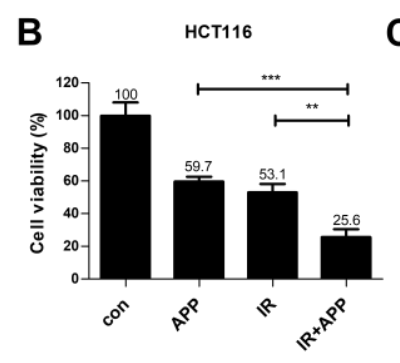

C
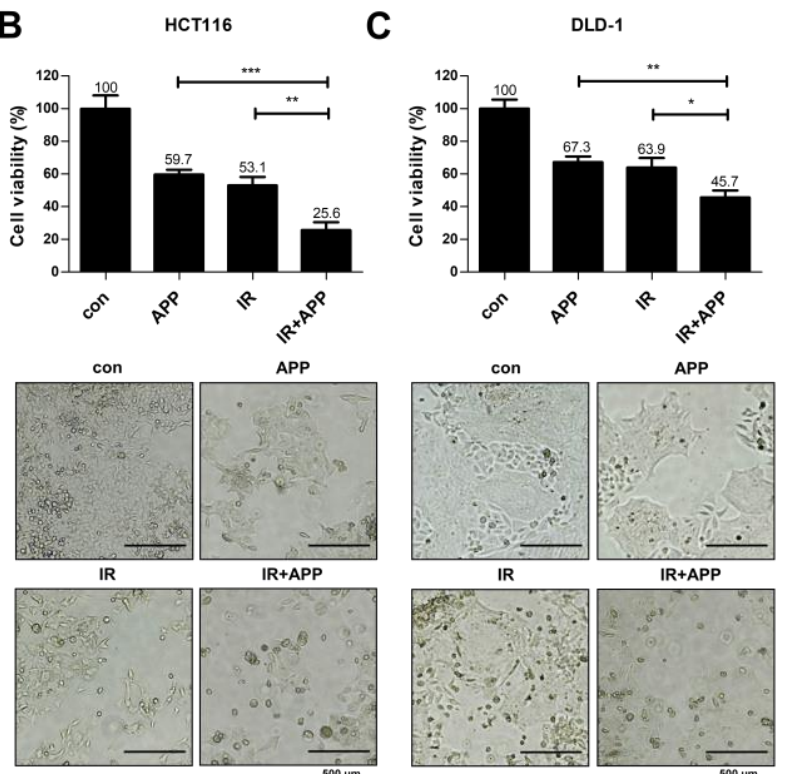
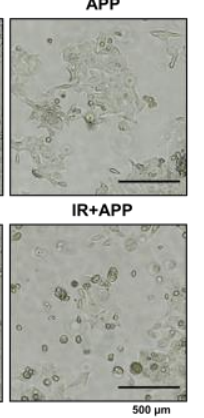
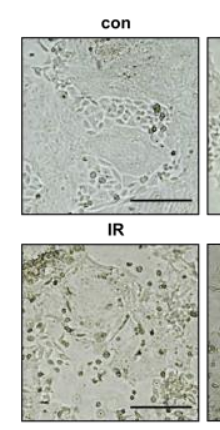

APP
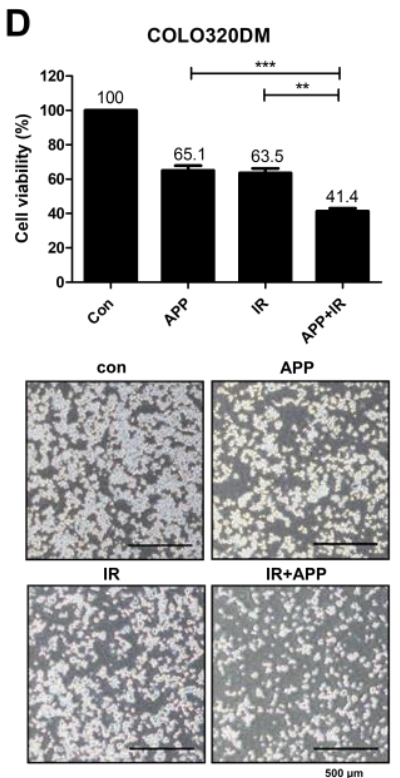

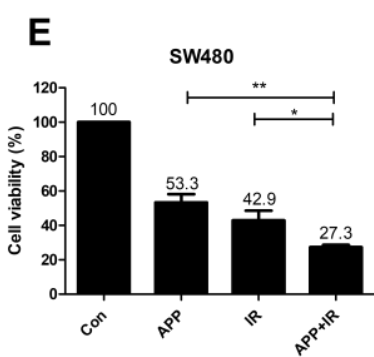

con

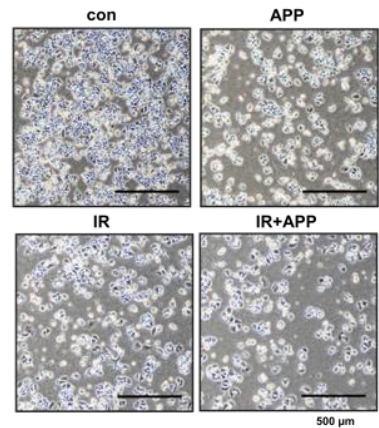

Figure 2. Radiosensitizer effect of APP. (A) Clonogenic assays. Clonogenic assays on DLD-1 and HCT116 cells were performed as described in the Materials and Methods. 'DMSO', DMSO-treated mock control; 'APP', cells treated with $7.5 \mathrm{nM}$ APP. (B-E) Cell counting assay. Cell counting assays were performed as described in the Materials and Methods. 'Con', DMSO-treated mock control cells; 'APP 7.5 nM', cells treated with $7.5 \mathrm{nM}$ APP; 'IR 3Gy', cells treated with 3Gy IR; 'IR+APP', cells treated with 7.5 nM APP and 3Gy IR. The lower panel shows microscopic images prior to cell detachment. Experiments are repeated in triplicate and results indicate the mean of triplicate assays [22, 28]. Each bar in pictures indicates $500 \mu \mathrm{m}$. (F) Immunoblot assay for $\gamma \mathrm{H} 2 \mathrm{AX}$. HCT116, DLD-1, SW480 and COLO320DM cells were incubated with or without 7.5 nM APP, exposed to 3 Gy IR, and incubated for $72 \mathrm{~h}$ prior to harvest. Scale bar: $500 \mu \mathrm{m}$.

Table 1. DER analysis. 


\section{$7.5 \mathrm{nM}$}

\section{HCT116}

DLD-1
1.13

1.31

Note: DER (Dose-Enhancement Ratio) values were calculated from the clonogenic assay (Fig. 2A). Radiation doses at a survival fraction of 0.1 of mock control or $7.5 \mathrm{nM}$ APP-treated group were acquired for HCT116 cells as 3.16 and $2.79 \mathrm{~Gy}$, and DLD-1 as 3.28 and $2.51 \mathrm{~Gy}$, respectively. Using these doses, DER values were calculated as follows: radiation doses at a survival fraction of 0.1 of the IR-only treatment group/radiation doses at a survival fraction of 0.1 of the APP treated group.

\subsection{Combined treatment with APP and IR increases apoptosis}

To identify the cell death pathway induced by APP radiosensitization, we first assessed whether combined treatment with APP and IR could induce apoptosis in HCT-116 and DLD-1 cells (Fig. 3A). Using an Annexin V-PI apoptosis detection kit I, we found that apoptotic cell death in both CRC cell lines was more than 2-fold greater following combined treatment with APP and IR than after treatment with APP or IR alone. Immunoblot analyses also showed that the levels of cleaved caspase-3, caspase-9, and PARP were higher in cells treated with combination of APP and IR compared with those separately treated with APP or IR alone (Fig. 3B). Immunoblot analysis of the mitochondrial and cytosol fractions of these cells also showed that the concentration of cytochrome $c$ in the cytosol was higher following treatment with APP and IR than with either alone (Fig. 3C), indicating that combined treatment with APP and IR enhanced cell death by activating the apoptosis pathway.
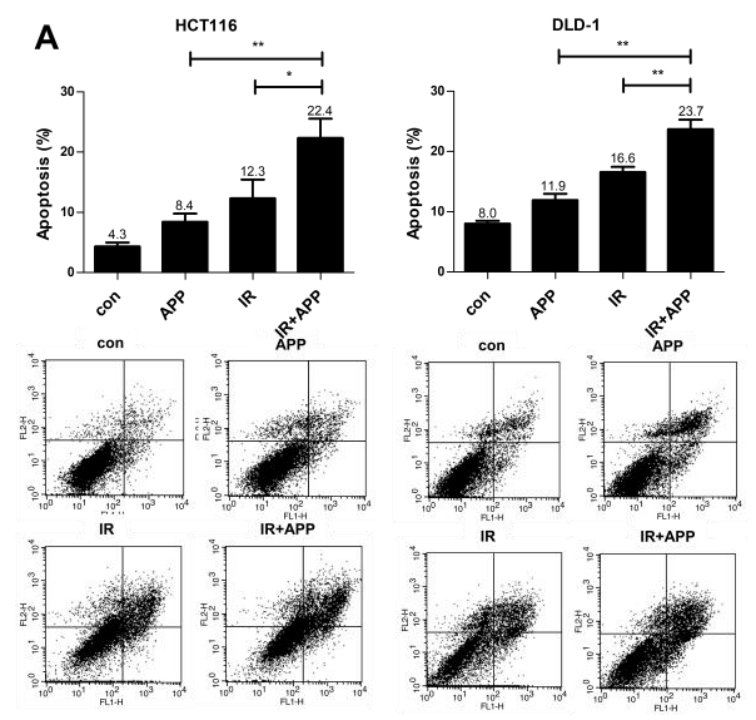

B

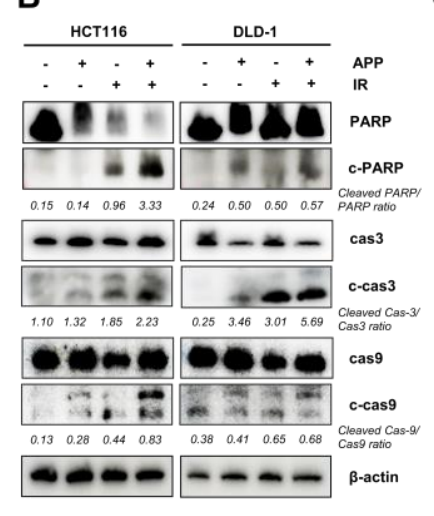

C

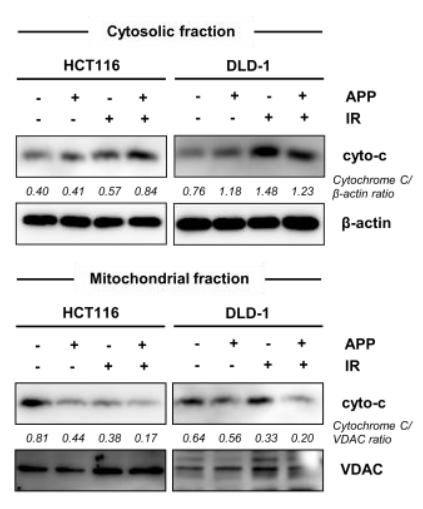

Figure 3. Induction of apoptotic cell death by combined treatment with APP and IR in CRC cells. (A) Annexin V-PI assay for detection of apoptosis. HCT116 and DLD-1 cells were treated with 7.5 nM APP alone, 3 Gy IR alone, or both for $72 \mathrm{~h}$. The assay method is described in the Materials and Methods. The lower panel shows representative FACSort flow cytometry images prior to quantitative analyses. 'Con', DMSO-treated mock control cells; 'APP $7.5 \mathrm{nM}^{\prime}$, cells treated with $7.5 \mathrm{nM}$ APP; 'IR 3Gy', cells treated with 3Gy IR; 'IR+APP', cells treated with $7.5 \mathrm{nM}$ APP and 3Gy IR. Experiments are repeated in triplicate and results indicate the mean of triplicate assays. (B) Immunoblot assays. The assays for detecting caspase-3, caspase-9 and PARP activation in cells treated with APP and IR. 'cas3', caspase-3; 'cas9', caspase-9. 'c-cas3', 'c-cas9' and 'c-PARP' indicate the cleaved forms of caspases-3 and -9 and PARP. HCT116 and DLD- 1 cells $\left(1 \times 10^{5}\right)$ were incubated with or without $7.5 \mathrm{nM}$ $\mathrm{APP}$, exposed to $3 \mathrm{~Gy}$ IR and incubated for $72 \mathrm{~h}$ prior to harvest. (C) Immunoblot assays. Immunoblot assay for 
detecting release of cytochrome $c$ from mitochondria to cytosol in cells treated with APP and IR. HCT116 and DLD-1 cells $\left(3 \times 10^{5}\right)$ were incubated with or without $7.5 \mathrm{nM}$ APP for $16 \mathrm{~h}$, and then exposed to 3 Gy IR for $72 \mathrm{~h}$.

\subsection{Radiosensitizing effect of APP is mediated by intracellular ROS accumulation}

Because the disruption of ROS homeostasis has been shown to be a main cause of radiosensitizer-induced apoptosis in our previous studies [27, 28], we also performed H2DCFDA-based intracellular ROS detection assays. These assays showed that the production of ROS was 1.3 1.5 fold higher in cells treated with APP and IR than in cells treated with APP or IR alone (Fig. 4A). Treatment with the ROS scavenger NAC abrogated the increase in ROS production induced by combined treatment of APP and IR (Fig. 4B). To test whether the NAC-associated reduction in ROS reduces cell death following combined treatment with APP and IR, cell counting and Annexin V-PI apoptosis assays were performed, both of which showed that NAC pre-treatment prior to combined treatment with APP and IR inhibited the induction of cell death (Fig. 5A and 5B). Immunoblot analyses also showed that NAC pre-treatment reduced $\gamma \mathrm{H} 2 \mathrm{AX}$ expression, the release of cytochrome $c$ into cytosol, and the cleavages of PARP and caspases induced by combined treatment with APP and IR (Fig 6A-C). These findings indicate that the DNA damage and cell death induced by combined treatment with APP and IR were due to the accumulation of ROS in both CRC cell lines.
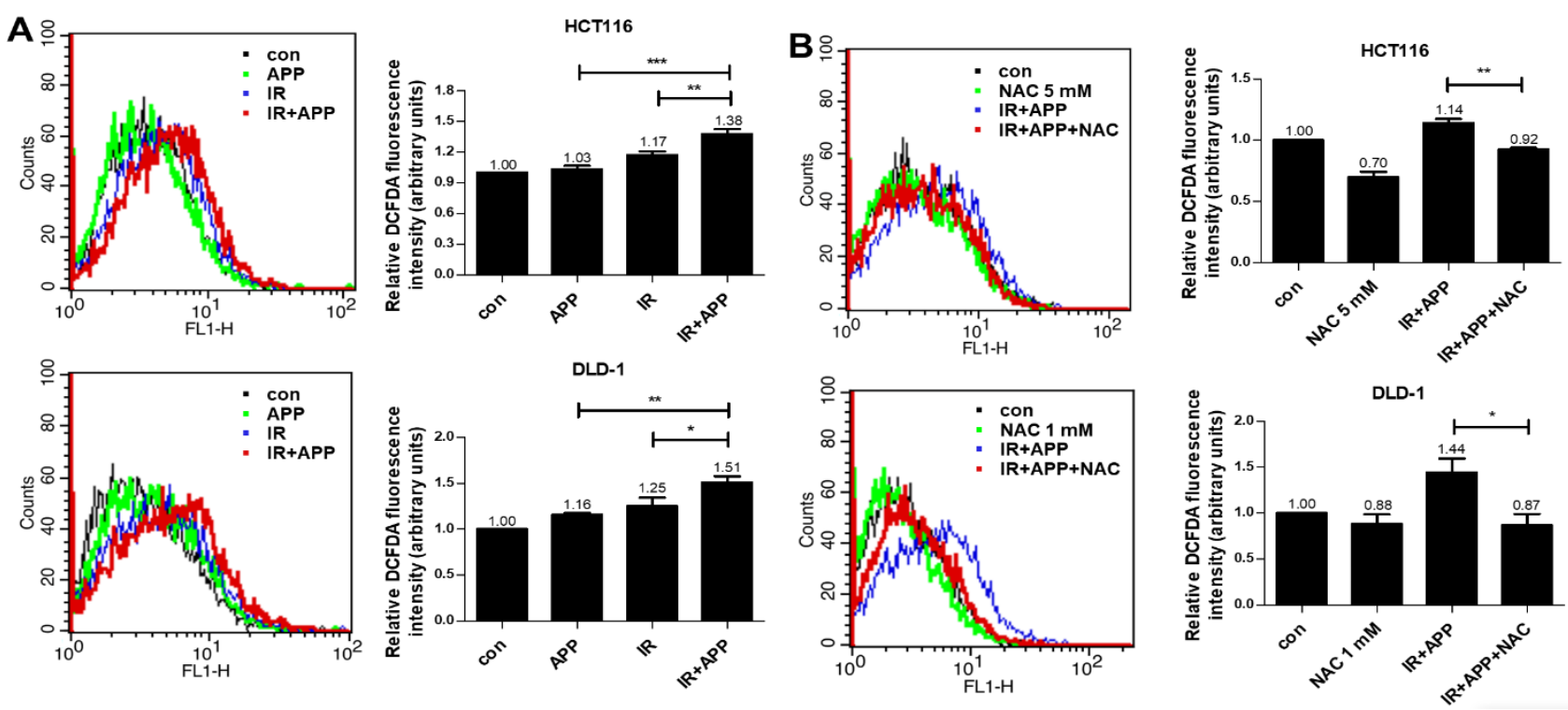

Figure 4. Enhancement of ROS accumulation in CRC cells treated with APP and IR. (A) Detection of ROS production. ROS detection in cells treated with APP alone, IR alone, or both by FACSort flow cytometry. HCT116 and DLD- 1 cells $\left(5 \times 10^{5}\right)$ were treated with or without $7.5 \mathrm{nM}$ APP for $16 \mathrm{~h}$, and then exposed to 3 Gy IR for $24 \mathrm{~h}$ prior to harvesting. 'Con', DMSO-treated mock control cells; 'APP', cells treated with $7.5 \mathrm{nM}$ APP; 'IR', cells treated with 3Gy IR; 'IR+APP', cells treated with both $7.5 \mathrm{nM}$ APP and 3Gy IR. Experiments are repeated in triplicate and results indicate the mean of triplicate assays. (B) Effects of NAC on ROS production. HCT116 cells were pre-treated with $5 \mathrm{mM} \mathrm{NAC}$ for $2 \mathrm{~h}$, and DLD-1 cells were pre-treated with $1 \mathrm{mM} \mathrm{NAC}$ for $1 \mathrm{~h}$, before treatment with APP, IR, or both. 'Con', DMSO-treated mock control cells; 'APP', cells treated with 7.5 nM APP; 'IR', cells treated with 3Gy IR; 'IR+APP', cells treated with $7.5 \mathrm{nM}$ APP and 3Gy IR; 'IR+APP+NAC', cells pre-treated with NAC and treated with $7.5 \mathrm{nM}$ APP and 3Gy IR. Experiments are repeated in triplicate and results indicate the mean of triplicate assays. 


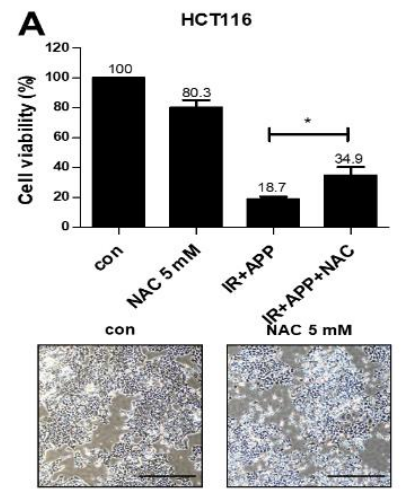

IR+APP

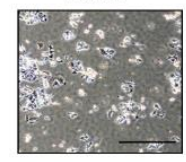

IR+APP+NAC

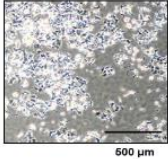

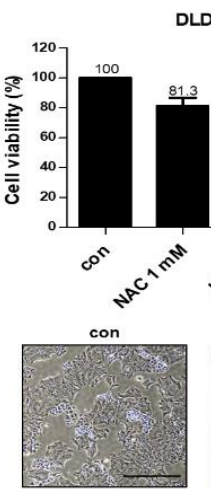

IR+APP

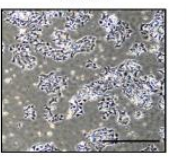

LD-1
B
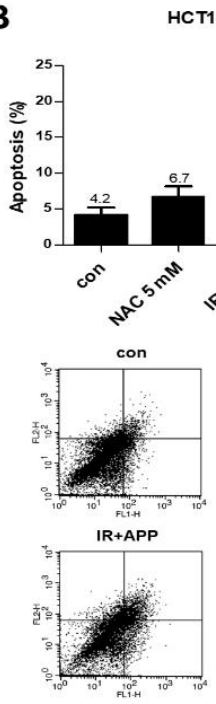

CT116
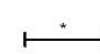

$\stackrel{18.5}{1.5}$ 5
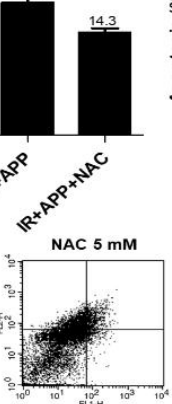

IR+APP+NAC

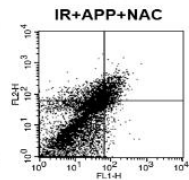

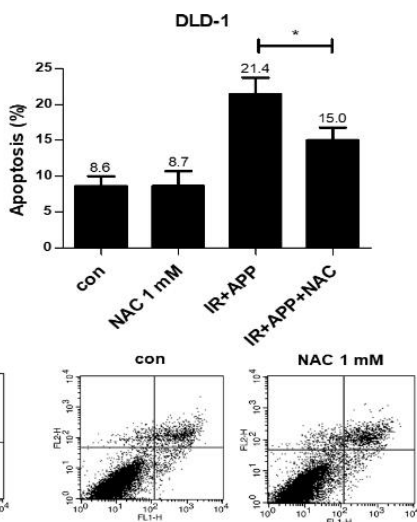

$18+A P P+N A C$
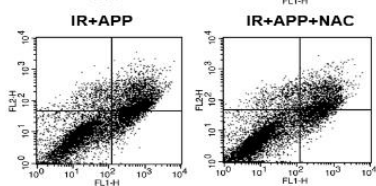

Figure 5. Effect of ROS on radiosensitizing role of APP. (A) Cell counting assay. HCT116 or DLD- 1 cells $\left(5 \times 10^{5}\right)$ were treated with or without 7.5 nM APP, and then exposed to 3 Gy IR for $24 \mathrm{~h}$ prior to harvest. In addition, HCT116 cells were pre-treated with $5 \mathrm{mM} \mathrm{NAC} \mathrm{for} 2 \mathrm{~h}$ and DLD- 1 cells were treated with $1 \mathrm{mM} \mathrm{NAC} \mathrm{for} 1 \mathrm{~h}$, before combined treatment with APP and IR. 'Con', mock control cells; 'NAC', cells pre-treated with 5 or $1 \mathrm{mM}$ NAC; 'IR+APP'; cells treated with $7.5 \mathrm{nM}$ APP and 3Gy IR; 'IR+APP+NAC', cells pre-treated with NAC and treated with 7.5 nM APP and 3Gy IR. Each lower panel shows representative microscopic images prior to cell detachment. Bars in cell pictures indicate $500 \mu \mathrm{m}$. Experiments are repeated in triplicate and results indicate the mean of triplicate assays. (B) Annexin V-PI assay for detection of apoptosis. Samples were prepared as described in (A), and apoptosis rates for each condition were determined by flow cytometry. Experiments are repeated in triplicate and results indicate the mean of triplicate assays. Each lower panel indicates representative FACSort flow cytometry images prior to quantitative analyses. Scale bar: $500 \mu \mathrm{m}$.
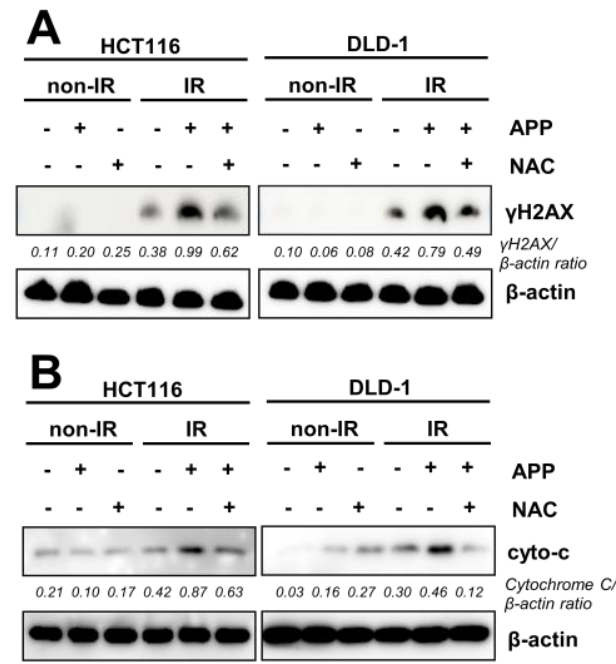

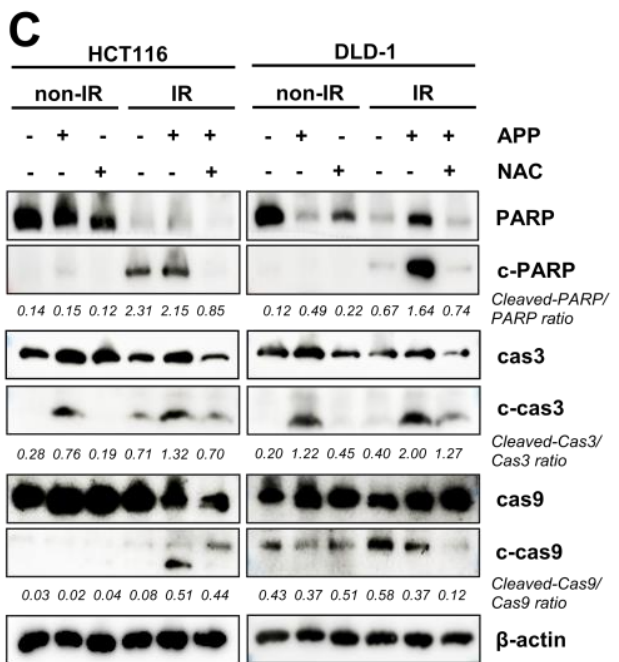

Figure 6. Effect of NAC on the activation of cell death-related proteins. HCT116 or DLD- 1 cells $\left(5 \times 10^{5}\right)$ were pre-treated with $5 \mathrm{mM} \mathrm{NAC} \mathrm{for} 2 \mathrm{~h}$ or with $1 \mathrm{mM}$ for $1 \mathrm{hr}$, respectively, followed by treatment with or without $7.5 \mathrm{nM}$ APP, 3 Gy IR, or both. Immunoblot assays were performed for the detection of $(\mathbf{A}) \gamma \mathrm{H} 2 \mathrm{AX}$ activation, (B) cytochrome $c$ release, and $(C)$ apoptosis proteins. Activated caspase-3, caspase- 9 and PARP were detected in cells pre-treated with NAC and treated with APP and IR for 72 h. 'cas3', caspase-3; 'cas9', caspase-9; 'c-cas3', cleaved caspase-3; 'c-cas9', cleaved caspase-9; 'c-PARP', cleaved PARP.

\subsection{In vivo radiosensitization effect of $A P P$}

Finally, we tested whether APP radiosensitized tumors by inducing apoptosis in vivo. HCT116 cells were injected into Balb-c nude mice, and xenograft tumors were treated intra-tumorally with $5 \mathrm{mg} / \mathrm{kg}$ APP, $5 \mathrm{~Gy} \mathrm{IR}$, both or neither. 
Each xenograft tumor was harvested 30 days later and TUNEL assays were performed to detect apoptotic cells (Fig. 7A). Measurements of the percentages of cells that had undergone apoptosis in each group (Fig. 7A, right panel) and the relative ratios of stained (apoptotic) areas (Fig. 7A, left panel) showed that the percentages of dead cells in the control, APP-treated, IR-treated, and APP+IR treated tissue samples were $4.9 \%, 26.1 \%, 50.2 \%$, and $74.2 \%$, respectively, with statistical analyses showing that combined treatment with APP and IR enhanced apoptosis compared with treatment with APP or IR alone. In addition to showing that APP could enhance apoptosis in vivo; these findings showed that APP could induce apoptosis in vitro and in vivo by enhancing DNA damage and ROS production in CRC cells (Fig. 7B).

A

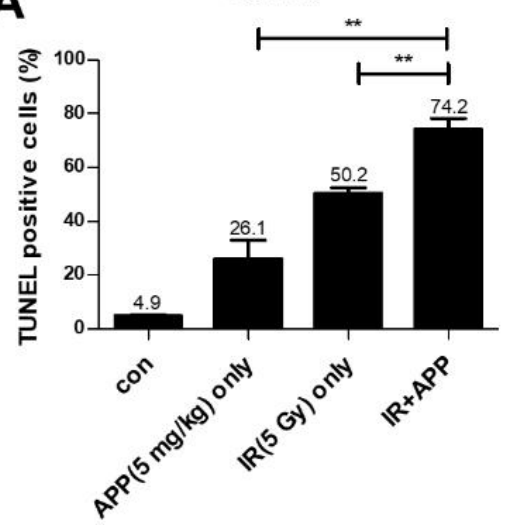

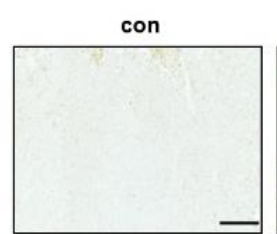

IR(5 Gy) only

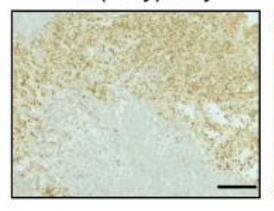

$\operatorname{APP}(5 \mathrm{mg} / \mathrm{kg})$ only

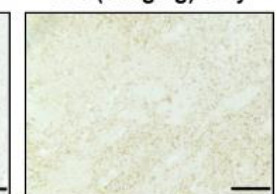

IR+APP

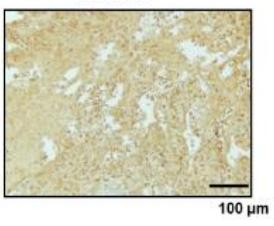

B

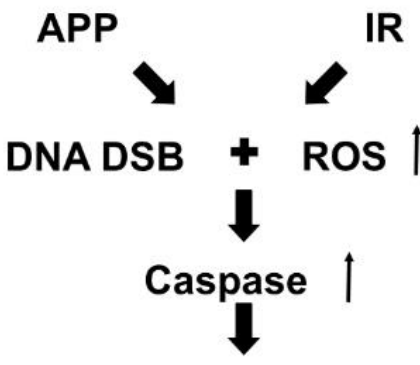

Apoptosis

Figure 7. In vivo radiosensitizing effects of APP. (A) TUNEL assays measuring apoptotic cells in mouse xenografts. Mice injected with HCT116 cells were divided into four groups, a control group, an APP only group (5 $\mathrm{mg} / \mathrm{kg}$ ), an IR only group (5 Gy), and an APP+ IR group, in which mice were pretreated with $5 \mathrm{mg} / \mathrm{kg} \mathrm{APP} \mathrm{for} 6$ $\mathrm{h}$ prior to IR irradiation. Each group contained 5 mice, and apoptotic cells in each xenograft were detected by TUNEL assays. The dark brown area in each tissue sample, indicating staining with TUNEL reagent, was detected using Image J software. The graph indicates quantitative analyses of the ratios of TUNEL-stained apoptotic cells to total cells. 'Con', mock control; 'APP only', mice treated with $5 \mathrm{mg} / \mathrm{kg} \mathrm{APP}$; 'IR only', mice treated with 5 Gy IR; 'IR+APP’, mice treated with $5 \mathrm{mg} / \mathrm{kg}$ APP and 5 Gy IR. Each bar in tissue figures indicates $100 \mu \mathrm{m}$. Experiments are repeated in triplicate and results indicate the mean of triplicate assays. (B) Schematic diagram showing the radiosensitization effect of APP. Scale bar: $100 \mu \mathrm{m}$.

\section{Discussion}

In this study, we detected APP induced the expression of phospho- $\mathrm{H} 2 \mathrm{AX}$ $(\gamma \mathrm{H} 2 \mathrm{AX})$, a major biomarker for the DNA damage response (DDR) responding several DNA damage inducers such as IR that can recruit DNA repair proteins [29-31]. H2AX is a component of histone proteins in chromatin that is rapidly phosphorylated at the serine-139 position by ataxia telangiectasia mutated (ATM) and ATM-Rad3-related (ATR), yielding $\gamma \mathrm{H} 2 \mathrm{AX}$, in response to various DNA damaging reagents $[32,33]$. The induction of $\gamma \mathrm{H} 2 \mathrm{AX}$ by APP suggests that APP might damage DNA in CRC cells. We also found that APP induced radio-sensitization of CRC cells. Both clonogenic and cell counting assays showed that APP enhanced IR-induced cell growth retardation, with the combined treatment of APP and IR also increasing $\gamma \mathrm{H} 2 \mathrm{AX}$ induction. These results showed that APP may be a candidate radiosensitizer as well as a candidate anti-cancer drug against CRC cells. The combined treatment of APP and IR also enhanced apoptosis in HCT116 and DLD-1 CRC cells by inducing the release of mitochondrial cytochrome $c$ into the cytosol. We also observed that in vivo treatment of tumor xenografts with the combined treatment of APP and IR enhanced apoptotic cell death more than 3-fold. These results indicated that the combined treatment of APP and IR enhanced the apoptosis induction. Two major pathways 
of apoptosis have been identified: the extrinsic and intrinsic pathways [34, 35]. The extrinsic pathway begins with death receptor/ligand binding [36, 37] and proceeds through activation of the initiator caspases- 8 and -10 . Activated cleaved caspases sequentially induce the cleavage of the effector caspases- 3 and -7 , inducing apoptosis [33]. The intrinsic pathway is initially induced by intracellular stresses, such as DNA damage, ER stress, hypoxia and metabolic stress. These stresses lead to changes in mitochondrial outer membrane permeabilization and the release of cytochrome $c$, which interacts with apoptotic protease activating factor 1 (APAF1), thereby inducing apoptosome assembly and the activation of caspase-9 $[38,39]$. Active caspase- 9 continuously activates caspases-3 and -7 to induce apoptosis. Caspases- 8 and -9 act as initiator caspases in the extrinsic and intrinsic pathways, respectively, resulting in the activation of caspase-3, a common 'executioner' caspase in both apoptotic pathways. The BH3-only protein $\mathrm{BH} 3$-interacting death domain agonist (BID) provides crosstalk between the extrinsic and intrinsic apoptotic pathways by inducing caspase- 8 cleavage [40]. Our immunoblotting data results demonstrate the activation of caspases- 3 and -9 and the release of mitochondrial cytochrome $c$, results consistent with the involvement of the intrinsic apoptosis pathway. Because activation or modulation of apoptosis-related proteins, such as p53, p21, Bcl-2, Bax and caspases, constitute one of the strategies for radiotherapy-induced cancer elimination, therapeutic reagents that enhance the effects of radiotherapy should also target the apoptosis machinery in cells [41-43]. Moreover, we found that the combined treatment of APP and IR increased ROS production, whereas treatment with the ROS scavenger NAC decreased ROS induction. Enhanced ROS was associated with enhanced apoptotic cell death, accompanied by $\gamma \mathrm{H} 2 \mathrm{AX}$ induction and cytochrome $c$ release. These three effects of APP and IR - apoptosis, DNA damage and mitochondrial outer membrane disruption - were likely due to ROS production, as these effects were reversed by treatment with NAC. ROS may be an especially important mediator of the radiosensitization effects of various regents, as well as APP, in several types of cancer $[22,27,28]$. Radiosensitizers identified to date include small molecules, such as free radicals and pseudosubstrates; nanomaterials, including gold-based nanometallic materials with flexible surface engineered molecular structures and favorable kinetic properties that promote radiosensitization; and macromolecules such as miRNAs, peptides, proteins, and oligonucleotides. Several small molecule chemicals that enhance free radical production, such as APP, have been developed as radiosensitizers [44]. The therapeutic effect of conventional radiation treatment is usually mediated by the indirect action of free radicals produced by the radiolysis of water, followed by the destruction of biomolecules. These effects can be enhanced by small molecules that promote free radical production [45]. Taken together, these findings indicate that APP is a promising radiosensitizer candidate that induces apoptosis via DNA injury and the production of free radicals such as ROS.

\section{Materials and Methods}

\subsection{Cell cultures and Chemical reagents}

HCT116, DLD-1, SW480 and COLO320DM cell lines were purchased from the American Type Culture Collection (Rockville, MD, USA). Both cell lines were cultured in RPMI 1640 media (Corning, Manassas, VA, USA) supplemented with 10\% fetal bovine serum (FBS; Gibco, Grand Island, NY, USA) and 1\% streptomycin/penicillin (Wellgene, Gyeongsan-si, South Korea) at $37^{\circ} \mathrm{C}$ in a $5 \%$ $\mathrm{CO}_{2} \quad$ incubator. $\quad \beta$-apopicropodophyllin (5-(3,4,5-trimethoxyphenyl)furo(3',4':6,7)naphtho(2,3-d)(1,3)dioxol-6(9H)-one)) was synthesized by J\&C Sciences (Daejeon, South Korea). 


\subsection{MTT Assay and IC50 determination}

HCT116, DLD-1, SW480 and COLO320DM cells were seeded on 96 well plates $\left(3 \times 10^{3}\right.$ cells/well $)$ and incubated with various concentrations $(6.25,12.5,25$, $50,100 \mathrm{nM})$ of APP for 48 or $72 \mathrm{~h}$ at $37^{\circ} \mathrm{C}$. A $20 \mu \mathrm{L}$ aliquot of MTT (3-(4,5-dimethylthiazol-2-yl)-2,5-diphenyltetrazolium bromide) solution (2 $\mathrm{mg} / \mathrm{mL}$ ) was added to each well, and the cells incubated for $1 \mathrm{~h}$ at $37^{\circ} \mathrm{C}$. Formazan crystals generated in living cells were dissolved in $100 \mu \mathrm{L}$ of DMSO, and the absorbance of individual wells at $545 \mathrm{~nm}$ was measured using a microplate reader (Molecular Devices, San Jose, CA, USA). The 50\% inhibitory concentration (IC50) of APP was calculated by concentration-response analysis using Softmax Pro software (Molecular Devices, Sunnyvale, CA, USA).

\subsection{Clonogenic assay}

HCT116 and DLD-1 cells were seeded in $60 \mathrm{~mm}$ dishes at concentrations that yielded 20-100 colonies/dish (100, 200, 400, 600, 1000 cells/dish). After incubation for $24 \mathrm{~h}$, the cells were treated with or without $7.5 \mathrm{nM}$ APP for $16 \mathrm{~h}$ and irradiated with 1, 2, 3, or 4 Gy ${ }^{137} \mathrm{Cs}$ as a source of $\gamma$-ionizing radiation (Atomic Energy of Canada, Ltd., Mississauga, ON, Canada). The cells were incubated for a maximum of 14 days until colonies formed, and colonies larger than $200 \mu \mathrm{m}$ in diameter were stained with $1 \%$ methylene blue in methanol. Stained colonies were counted using a colony counter (Imaging Products, Chantilly, VA, USA). The number of colonies per dish was calculated relative to the number of cells seeded per dish, and the dose-enhancement ratio (DER) of each cell line was determined from the numbers of colonies using Excel software (Microsoft Co., Redmond, WA, USA).

\subsection{Cell counting assay}

HCT116, DLD-1 cells, SW480 and COLO320DM $\left(1 \times 10^{5}\right.$ cells/60 mm dish) were pre-treated with or without $7.5 \mathrm{nM} \mathrm{APP}$ for $16 \mathrm{~h}$ and then exposed to $3 \mathrm{~Gy}$ irradiation. The cells were incubated for $72 \mathrm{~h}$ at $37^{\circ} \mathrm{C}$, collected by trypsinization, washed twice with cold PBS, and stained with trypan blue. The cells were counted using an EVETM Automated Cell Counter (NanoEntek, Seoul, South Korea). Results are reported as the mean of triplicate assays.

\subsection{Immunoblot analysis}

Harvested cells were lysed with RIPA buffer (50 mM Tris-HCl, pH 7.6, $150 \mathrm{mM}$ $\mathrm{NaCl}, 1 \%$ Triton X-100, 1\% sodium deoxycholate, 0.1\% SDS, 2 mM EDTA), and the lysates were centrifuged at $14,000 \mathrm{~g}$ for $20 \mathrm{~min}$. The supernatants were removed, and the concentrations of protein measured at $280 \mathrm{~nm}$ using Bradford solution (Bio-Rad, Hercules, CA, USA) and a microplate reader (Molecular Devices). A $20 \mathrm{mg}$ aliquot of lysate from each well was loaded onto an SDS-PAGE gel, electrophoresed, and transferred to a nitrocellulose membrane. After blocking for $1 \mathrm{hr}$ with $5 \%$ skim milk, the membranes were incubated at $4^{\circ} \mathrm{C}$ with primary antibody in $5 \%$ BSA solution at $4^{\circ} \mathrm{C}$, followed by washing and incubation with secondary antibody for $1 \mathrm{hr}$ at room temperature. Bands were detected with Clarity ${ }^{\mathrm{TM}}$ Western ECL Substrate (Bio-Rad) and visualized with Amersham ImageQuant 800 (GE Healthcare Bio-Sciences Corp., Marlborough, MA, USA). The primary antibodies used in this study included antibodies to pro-caspase-3, pro-caspase-9, cleaved caspase-3, cleaved caspase-9, pro-PARP 
and cleaved PARP (Cell Signaling Technology, Beverly, MA, USA). An anti- $\beta$-actin antibody (Sigma-Aldrich, St. Louis, MO, USA) was used as a loading control. The relative band densities were determined via densitometry using ImageJ software (NIH, Bethesda, USA) and then normalized to that of each control. All experiments were repeated twice and calculated to mean values. Bands in the figures signify representative data.

\subsection{Isolation of mitochondrial and cytosolic fractions}

HCT116 and DLD-1 cells were pre-treated with $7.5 \mathrm{nM}$ APP and irradiated at $3 \mathrm{~Gy}$, followed by incubation for $48 \mathrm{~h}$ at $37^{\circ} \mathrm{C}$. The cells were incubated with trypsin-EDTA for $5 \mathrm{~min}$, collected by centrifugation at $250 \mathrm{~g}$ for $1 \mathrm{~min}$, resuspended in extraction buffer ( $1 \mathrm{M}$ sucrose, $1 \mathrm{M}$ HEPES, pH 7.4, $1 \mathrm{M} \mathrm{KCl}, 1 \mathrm{M}$ $\mathrm{MgCl} 2,0.25 \mathrm{M}$ EGTA, and $1 \mathrm{M}$ DTT), homogenized, and centrifuged at $12000 \mathrm{~g}$ at $4{ }^{\circ} \mathrm{C}$ for $15 \mathrm{~min}$. The supernatants were regarded as the cytosolic fractions. Each pellet was resuspended in lysis buffer $(50 \mathrm{mM}$ Tris- $\mathrm{HCl}, \mathrm{pH} 7.6,150 \mathrm{mM} \mathrm{NaCl}$, $1 \%$ Triton X-100, $1 \%$ sodium deoxycholate, $0.1 \%$ SDS, 2 mM EDTA) and regarded as the mitochondrial fraction. The fractions were immunoblotted with an anti-cytochrome $c$ antibody, with VDAC used as a loading control. These experiments were performed in triplicate.

\subsection{Annexin V-Propidium iodide assay}

HCT116 and DLD- 1 cells $\left(1 \times 10^{5}\right.$ cells $/ 60 \mathrm{~mm}$ dish $)$ were treated with or without $7.5 \mathrm{nM}$ APP and exposed to 3 Gy IR. The cells were incubated for $72 \mathrm{~h}$, collected by trypsinization, washed twice with cold PBS, and stained with FITC Annexin V apoptosis detection kit I reagent (Becton Dickinson, Franklin Lakes, NJ, USA), as described in the manufacturer's protocol. Samples were loaded onto a FACSort flow cytometer (Becton Dickinson), and the fraction of apoptotic cells was measured ( $x$-axis, FL1 channel; $y$-axis, FL-2 channel). These experiments were repeated in triplicate.

\subsection{ROS detection}

HCT116 and DLD- 1 cells $\left(5 \times 10^{5}\right.$ cells $/ 60 \mathrm{~mm}$ dish) were pre-treated with or without $7.5 \mathrm{nM}$ APP, exposed to 3 Gy IR, and incubated for $24 \mathrm{~h}$. The cells were subsequently stained with $25 \mathrm{mM}$ H2DCFDA (Merck, Darmstadt, Germany) for 5 min, trypsinized, harvested by centrifugation and resuspended in cold PBS. Samples were loaded onto a FACSort flow cytometer (Becton Dickinson) for detection and analysis of intracellular ROS ( $x$-axis, FL1 channel; $y$-axis, Counts).

\subsection{TUNEL assay of xenograft}

The protocols of all animal experiments were approved by the Institutional Animal Care and Use Committee (IACUC No. kirams 2019-0064). Xenografts were generated by subcutaneously injecting $1 \times 10^{7}$ HCT116 cells/mouse into 6-week-old BALB/cAnNCrj-nu/nu mice (Envigo, Cambridgeshire, UK). The mice were divided into four groups of four mice each, with one group each treated with APP alone, IR alone, APP+IR, and no treatment (control). When xenografts reached $100-120 \mathrm{~mm}^{3}$ in size, $5 \mathrm{mg} / \mathrm{kg}$ APP in DMSO were intratumorally injected into mice in the APP only and APP+IR groups, with an equal volume of DMSO (vehicle) intratumorally injected into mice in the IR only and control groups. Six hours later, mice in the IR only and APP+IR groups were locally irradiated with 5 Gy IR using a ${ }^{60} \mathrm{Co} \gamma$-ray source (Theratron 780; AECL Ltd., Mississauga, ON, Canada). Treatments were repeated three times at 3 day 
intervals for a total of 12 days. The mice were sacrificed 30 days after the start of the experiment. Extracted tumors were fixed with formaldehyde, embedded in paraffin and sectioned. TUNEL assays for detection of dUTP nicks were performed by Super Bio Chips Co. (Seoul, South Korea). Each stained tissue sample was photographed with an Olympus BX 53 (Olympus, Shinjuku, Tokyo, Japan), and the ratios of TUNEL-positive to total cells in each image were measured with Image J software (NIH, Bethesda, MD, USA). The percentages of TUNEL-positive cells in each tissue sample were calculated relative to numbers of cells in the control group.

\subsection{Statistical analysis}

Results are reported as means \pm standard deviations (SDs), shown as error bars, and compared using Student's t-tests. All statistical analyses were performed using GraphPad Prism software (GraphPad Software, La Jolla, CA, USA), with P-values $<0.05$ considered statistically significant.

Author Contributions: N.-G. L, A.-R.K., and J.-H.K. performed the majority of the experiments, interpreted the data, and calculated the statistics. J.-Y.S., S.-G.H., and H.-D.U. contributed interpretation of the initial experiments and contributed to the writing of manuscript. J.K. and J.K.P. contributed conception and design of the study, manuscript writing, and financial support for conducting the research. All authors critically read and approved the final manuscript.

Funding: This research was supported by the Basic Science Research Program through the National Research Foundation of Korea (NRF) funded by the Ministry of Science, ICT (505312021), and by the Basic Science Research Program through the NRF (NRF-2021R1F1A1055981 and NRF-2020M2D9A2094153).

Competing interests: The authors declare that they have no competing interests.

Abbreviations

\begin{tabular}{cc}
\hline APP & $\beta$-apopicropodophyllin \\
CRC & Colorectal cancer \\
IR & $\gamma$-Ionizing radiation \\
ROS & Reactive oxygen species \\
PPT & Podophyllotoxin \\
Gy & Grey \\
DER & Dose-enhancement ratio \\
TUNEL & Terminal deoxynucleotidyl transferase dUTP nick end labeling \\
SD & Standard deviation \\
PI & Propidium iodide \\
NAC & N-acetylcysteine \\
\hline
\end{tabular}

\section{References}

1. Siegel, R.L.; Miller, K.D.; Sauer, A.G.; Fedewa, S.A.; Butterly, L.F.; Anderson, J.C.; Cercek, A.; Smith, R.A.; Jemal, A. Colorectal Cancer Statistics, 2020. CA Cancer J Clin. 2020, 70, 145-164.

2. Skuse, A. Constructions of Cancer in Early Modern England. Palgrave Macmillan, London, 2015, 20-39.

3. Mokhtari, R.B.; Homayouni, T.S.; Baluch, N.; Morgatskaya, E.; Kumar, S.; Das, B.; Yeger, H. Combination therapy in combating cancer. Oncotarget. 2017, 8, 38022-38043.

4. Xie, Y.-H.;Chen, Y.-X., Fang, J.-Y. Comprehensive review of targeted therapy for colorectal cancer. Signal Transduct Target Ther. 2020, 5, 22.

5. Banerjee, A.; Pathak, S.; Subramanium, V.D.; Dharanivasan, G.; Murugesan, R.; Verma, R.S. Strategies for targeted drug delivery in treatment of colon cancer: current trends and future perspectives. Drug Discovery Today 2017, 22, 1224-1232. 
6. Choi, J.Y.; Hong, W.G.; Cho, J.H.; Kim, E.M.; Kim, J.D.; Jung, C.H.; Hwang, S.G.; Um, H.D.; Park, J.K. Podophyllotoxin acetate triggers anticancer effects against non-small cell lung cancer cells by promoting cell death via cell cycle arrest, ER stress and autophagy. Int J Oncol. 2015, 47, 1257-1265.

7. Gordaliza, M.; García, P.A.; del Corral, J.M.M.; Castro, M.A.; Gómez-Zurita, M.A. Podophyllotoxin: distribution, sources, applications and new cytotoxic derivatives. Toxicon. 2004, 44, 441-459.

8. Hevener, K.; Verstak, T.A.; Lutat, K.E.; Riggsbee, D.L.; Mooney, J.W. Recent developments in topoisomerase-targeted cancer chemotherapy. Acta Pharm Sin B. 2018, 8(6), 844-861.

9. Delgado, J.L.; Hsieh, C.M.; Chan, N.L.; Hiasa, H. Topoisomerases as anticancer targets. Biochem J. 2018, 475(2), 373-398.

10. Champoux, J.J. DNA topoisomerases: structure, function, and mechanism. Annu Rev Biochem. 2001,70, 369-413.

11. Bush, N.G.; Evans-Roberts, K.; Maxwell, A. DNA topoisomerases. EcoSal Plus. 2015, 6(2).

12. Pommier, Y. Drugging topoisomerases: lessons and challenges. ACS Chem Biol. 2013, 8, 82-95.

13. Nitiss, J.L. Targeting DNA topoisomerase II in cancer chemotherapy. Nat Rev Cancer. 2009,9,338-350. 9. Leelaram, M.N.; Bhat, A.G.; Godbole, A.A.; Bhat, R.S.; Manjunath, R.; Nagaraja, V. Type IA topoisomera se

14. inhibition by clampclosure. FASEB J. 2013, 27,3030-3038.

15. Pommier, Y.; Leo, E.; Zhang, H.; Marchand, C. DNA topoisomerases and their poisoning by anticancer and antibacterial drugs. Chem Biol. 2010,17,421-433.

16. Hornyak, P.; Askwith, T.; Walker, S.; Komulainen, E.; Paradowski, M.; Pennicott, L.E.; Bartlett, E.J.; Brissett, N.C.; Raoof, A.; Watson, M.; Jordan, A.M.; Ogilvie, D.J.; Ward, S.E.; Atack, J.R.; Pearl, L.H.; Caldecott, K.W.; Oliver, A.W. Mode of action of DNA-competitive small molecule inhibitors of tyrosyl DNA phosphodiesterase 2. Biochem J. 2016, 473, 1869-1879.

17. Lindsey Jr., R.H.; Pendleton, M.; Ashley, R.E.; Mercer, S.L.; Deweese, J.E.; Osheroff, N. Catalytic core of human topoisomerase II $\alpha$ : insightsinto enzyme-DNA interactions and drug mechanism. Biochemistry 2014,53,6595-6602.

18. Gibson, E.G.; Deweese, J.E. Covalent poisons of topoisomerase II. Curr Top Pharmacol. 2013, 17, 1-12.

19. Olaussen, K.A.; Postel-Vinay, S. Predictors of chemotherapy efficacy in non-small-cell lung cancer: a challenging landscape. Ann Oncol. 2016, 27(11), 2004-2016.

20. Meisenberg, C.; Ashour, M.E.; El-Shafie, L.; Liao, C.; Hodgson, A.; Pilborough, A.; Khurram, S.A.; Downs, J.A.; Ward, S.E.; El-Khamisy, S.F. Epigenetic changes in histone acetylation underpin resistance to the topoisomerase I inhibitor irinotecan. Nucleic Acids Res. 2017, 45(3), 1159-1176.

21. Saif, M.W.; Kang, S.P.; Chu, E. Treatment of metastatic colorectal cancer: from cytotoxic agents to molecular agents and multitargeted strategies. Oncology (Williston Park). 2006, 14 Suppl 10, 11-19.

22. Kim, J.Y.; Cho. J.H.; Kim, E.M.; Shin, H.J.; Hwang, S.G.; Song, J.Y.; Um, H.D.; Park, J.K. $\beta$-apopicropodophyllin functions as a radiosensitizer targeting ER stress in non-small cell lung cancer. Biomed Pharmacother. 2019, 113, 108769.

23. Kim, J.Y.; Cho, J.H.; Choi, J.R.; Shin, H.J.; Song, J.Y.; Hwang, S.G.; Um, H.D.; Yoo, Y.D.; Kim, J.; Park, J.K. A novel anti-cancer role of $\beta$-apopicropodophyllin against non-small cell lung cancer cells. Toxicol Appl Pharmacol. 2018, 375, 39-49.

24. Li, Y.; Mao, Y.; Brandt-Rauf, P.W.; Williams, A.C.; Robert, L.F. Selective induction of apoptosis in mutant p53 premalignant and malignant cancer cells by PRIMA-1 through the c-Jun-NH2-kinase pathway. Mol Cancer Ther. 2005, 4, 901-909.

25. Hata, T.; Yamamoto, H.; Ngan, C.Y.; Koi, M.; Takagi, A.; Damdinsuren, B.; Yasui, M.; Fujie, Y.; Matsuzaki, T.; Hemmi, H., Xu, X.; Kitani, K.; Seki, Y.; Takemasa, I.; Ikeda, M.; Sekimoto, M.; Matsuura, N.; Monden, M. Role of p21waf1/cip1 in effects of oxaliplatin in colorectal cancer cells. Mol Cancer Ther. 2005, 4, 1585-1594.

26. Ahmed, D.; Eide, P.W., Eilertsen, I.A.; Danielsen, S.A.; Eknæs, M.; Hektoen, M.; Lind, G.E.; Lothe, R.A. Epigenetic and genetic features of 24 colon cancer cell lines. Oncogenesis. 2013, 2, e71.

27. Cho, H.J.; Ahn, K.C.; Choi, J.Y.; Hwang, S.G.; Kim, W.J.; Um, H.D.; Park, J.K. Luteolin acts as a radiosensitizer in non-small cell lung cancer cells by enhancing apoptotic cell death through activation of a p38/ROS/caspase cascade. Int J Oncol. 2015, 46, 1149-1158.

28. Hong, W.G.; Kim, J.Y.; Cho, J.H.; Hwang, S.G.; Song, J.Y.; Lee, E.A.; Chang, T.S.; Um, H.D.; Park, J.K. AMRI-59 functions as a radiosensitizer via peroxiredoxin I-targeted ROS accumulation and apoptotic cell death induction. Oncotarget 2017, 8, 114050-114064.

29. Albino, A.P.; Huang, X.; Jorgensen, E.; Yang, J.; Gietl, D.; Traganos, F.; Darzynkiewicz, Z. Induction of H2AX phosphorylation in pulmonary cells by tobacco smoke: a new assay for carcinogens. Cell Cycle 2004, 3, 1062-1068.

30. Ando, M.; Yoshikawa, K.; Iwase, Y.; Ishiura, S. Usefulness of monitoring gamma-H2AX and cell cycle arrest in HepG2 cells for estimating genotoxicity using a high-content analysis system. J Biomol Screen 2014, 19, 1246-1254.

31. Pouliliou, S.; Koukourakis, M.I. Gamma histone $2 \mathrm{AX}(\gamma-\mathrm{H} 2 \mathrm{AX})$ as a predictive tool in radiation oncology. Biomarkers 2014, 19, 167-180. 
32. Rogakou, E.P.; Pilch, D.R.; Orr, A.H.; Ivanova, V.S.; Bonner, W.M. DNA double-stranded breaks induce histone H2AX phosphorylation on serine 139. J Biol Chem. 1998, 273, 5858-5868.

33. Kinner, A.; Wu, W.; Staudt, C.; Iliakis, G. Gamma-H2AX in recognition and signaling of DNA double-strand breaks in the context of chromatin. Nucleic Acids Res. 2008, 36, 5678-5694.

34. Ichim, G.; Tait, S.W.G. A fate worse than death: apoptosis as an oncogenic process. Nat Rev Cancer 2016, 16, 539-548.

35. Carneiro, B.A.; El-Deiry, W.S. Targeting apoptosis in cancer therapy. Nat Rev Clin Oncol. 2020, 17: $395-417$.

36. Pennica, D.; Nedwin, G.E.; Hayflick, J.S.; Seeburg, P.H.; Derynck, R.; Palladino, M.A.; Kohr, W.J.; Aggarwal, B.B.; Goeddel, D.V. Human tumour necrosis factor: precursor structure, expression and homology to lymphotoxin. Nature 1984, 312, 724-729.

37. Pan, G.; O’Rourke, K.; Chinnaiyan, A.M.; Gentz, R.; Ebner, R.; Ni, J.; Dixit, V.M. The receptor for the cytotoxic ligand TRAIL. Science 1997, 276, 111-113.

38. Luo, X.; Budihardjo, I.; Zou, H.; Slaughter, C.; Wang, X. Bid, a Bcl2 interacting protein, mediates cytochrome c release from mitochondria in response to activation of cell surface death receptors. Cell 1998, 94, 481-490.

39. Tait, S.W.G.; Green, D.R. Mitochondria and cell death: outer membrane permeabilization and beyond. Nat Rev Mol Cell Bio 2010, 11, 621-632.

40. Li, H.; Zhu, H.; Xu, C.J.; Yuan, J. Cleavage of BID by caspase 8 mediates the mitochondrial damage in the Fas pathway of apoptosis. Cell 1998, 94, 491-501.

41. Maier, P.; Hartmann, L.; Wenz, F.; Herskind, C. Cellular Pathways in Response to Ionizing Radiation and Their Targetability for Tumor Radiosensitization. Int J Mol Sci. 2016, 17, 102.

42. Yasui, H.; Iizuka, D.; Hiraoka, W.; Kuwabara, M.; Matsuda, A.; Inanami, O. Nucleoside analogs as a radiosensitizer modulating DNA repair, cell cycle checkpoints, and apoptosis. Nucleosides Nucleotides Nucleic Acids 2020, 39, 439-452.

43. Buckley, A.M.; Lynam-Lennon, N.; O'Neill, H.; O'Sullivan, J. Targeting hallmarks of cancer to enhance radiosensitivity in gastrointestinal cancers. Nat Rev Gastroenterol Hepatol. 2020, 17, 298-313.

44. Wang, H.; Mu, X.; He, H.; Zhang, X.D. Cancer Radiosensitizers. Trends Pharmacol Sci. 2018, 39, 24-48.

45. Jiang, H.; Wang, H.; De Ridder, M. Targeting antioxidant enzymes as a radiosensitizing strategy. Cancer Lett. 2018, 438, 154-164. 